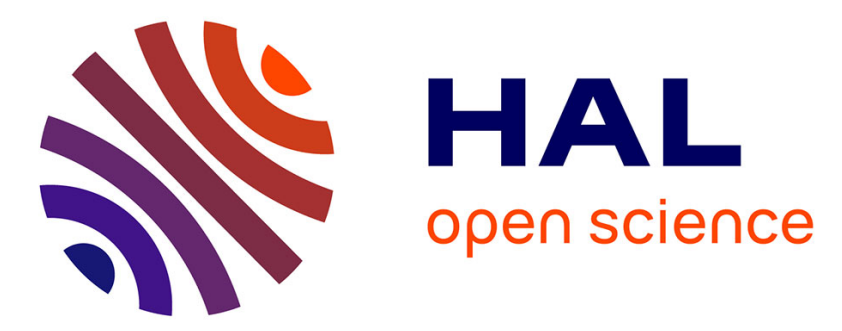

\title{
Normal stress measurements in sheared non-Brownian suspensions
}

\author{
S. Garland, G. Gauthier, Jérôme Martin, J. F Morris
}

\section{To cite this version:}

S. Garland, G. Gauthier, Jérôme Martin, J. F Morris. Normal stress measurements in sheared non-Brownian suspensions. Journal of Rheology, 2013, 57 (1), pp.71-88. 10.1122/1.4758001 . hal02941640

\section{HAL Id: hal-02941640 \\ https://hal.science/hal-02941640}

Submitted on 17 Sep 2020

HAL is a multi-disciplinary open access archive for the deposit and dissemination of scientific research documents, whether they are published or not. The documents may come from teaching and research institutions in France or abroad, or from public or private research centers.
L'archive ouverte pluridisciplinaire HAL, est destinée au dépôt et à la diffusion de documents scientifiques de niveau recherche, publiés ou non, émanant des établissements d'enseignement et de recherche français ou étrangers, des laboratoires publics ou privés. 
Normal stress measurements in sheared non-Brownian suspensions

S. Garland, ${ }^{1}$ G. Gauthier, ${ }^{1, a)}$ J. Martin, ${ }^{1, b)}$ and J. F. Morris ${ }^{2, c)}$

1) FAST, Université Paris Sud - 11, CNRS UMR 7608, bât. 502 Campus Universitaire 91405 Orsay cedex, France

2) Levich Institute and Dept. of Chemical Engineering; City College of New York; New York, NY 10031 USA

(Dated: 17 July 2012) 
Measurements in a cylindrical Taylor-Couette device of the shear-induced radial normal stress in a suspension of neutrally buoyant non-Brownian (non-colloidal) spheres immersed in a Newtonian viscous liquid are reported. The radial normal stress of the fluid phase was obtained by measurement of the grid pressure $P_{g}$, i.e. the liquid pressure measured behind a grid which restrained the particles from crossing. The radial component of the total stress of the suspension was obtained by measurement of the membrane pressure $P_{m}$, i.e. the total pressure obtained with a transducer exposed to both phases. Pressure measurements, varying linearly with the shear rate, were obtained for shear rates low enough to insure a grid pressure below a particle size dependent capillary stress. Under these experimental conditions, the membrane pressure is shown to equal the second normal stress difference, $N_{2}$, of the suspension stress whereas the difference between the grid pressure and the total pressure, $P_{g}-P_{m}$, equals the radial normal stress of the particle phase, $\Sigma_{r r}^{p}$. The collected data show that the radial normal stress is about one order of magnitude higher than the second normal stress difference of the suspension. The $\Sigma_{r r}^{p}$ values obtained in this manner are independent of the particle size, and their ratio to the suspension shear stress increases quadratically with $\phi$, in the range $0<\phi<0.4$. This finding, in agreement with the theoretical particle pressure prediction of Brady and Morris (J. Fluid Mech. 348 pp 103-139, 1997) for small $\phi$, supports the contention that the particle phase normal stress $\Sigma_{r r}^{p}$ is due to asymmetric pair interactions under dilute conditions, and may not require many-body effects. Moreover we show that the values of $\Sigma_{r r}^{p}$, normalized by the fluid shear stress, $\eta_{f}|\dot{\gamma}|$ with $\eta_{f}$ the suspending fluid viscosity and $|\dot{\gamma}|$ the magnitude of the shear rate, are well-described by a simple analytic expression recently proposed for the particle pressure.

\footnotetext{
a) Electronic mail: gauthier@fast.u-psud.fr

b)Electronic mail: martin@fast.u-psud.fr

c)Electronic mail: morris@ccny.cuny.edu
} 
Suspensions of non-Brownian particles are ubiquitous in a wide range of applications (concrete, water purification, "recycling" of nuclear waste) and have been the subject of many theoretical, numerical and experimental studies. Despite the large amount of work performed, the flowing properties of concentrated suspensions remain incompletely characterized. This work will address the measurement of normal stresses in a suspension, an area which has received limited attention.

By contrast, numerous studies over the past century have focused on the shear viscosity of particles immersed in a Newtonian fluid. Einstein (1905), in his pioneering work, calculated the viscosity of a dilute suspension (neglecting hydrodynamic interactions) of spheres as a function of the particle volume fraction $\phi: \eta=\eta_{0}(1+2.5 \phi)$. Batchelor and Green (1972), taking into account pair interaction, extended the result of Einstein to the second order: $\eta=\eta_{0}\left(1+2.5 \phi+B \phi^{2}\right)+\mathcal{O}\left(\phi^{3}\right)$ with $B=6.2$ for non-Brownian spheres. At higher concentration, multi-body interactions must be considered and numerous theoretical and empirical expressions for the shear viscosity with $\phi$ have been proposed (see the review of Stickel and Powell (2005)). While the various expressions tend to agree with the Einstein viscosity for dilute suspensions $(\phi<0.1)$, there is no consensus for the maximum packing volume fraction, $\phi_{\max }$, at which viscosity must diverge. A factor that complicates the viscosity measurement is shear-induced migration (Leighton and Acrivos (1987a,b)) which leads to non-uniform volume fraction of the sheared suspension (Phillips et al. (1992); Morris and Boulay (1999); Chow et al. (1994)). Gadala-Maria and Acrivos (1980) and Parsi and Gadala-Maria (1987) have shown that the viscosity is strongly influenced by the macroscopic spatial organization of the particles. In an attempt to eliminate shear-induced migration and measure the bulk viscosity of a suspension, Gauthier et al. (2005) used the damping of waves that propagate at the surface of a suspension, obtaining good agreement with several models but with $\phi_{\max }=0.58$. Recently, Ovarlez et al. (2006) used magnetic resonance imaging (MRI) to perform simultaneous measurement of shear stress, local shear rate and local volume fraction; this work found $\phi_{\max }=0.605$. Bonnoit et al. (2010), using an inclined plane rheometer, measured shear viscosity of non-Brownian suspensions up to similar solid fraction of $\phi=0.61$.

While the Newtonian viscosity of suspensions seems more or less understood, this is not the case for the non-Newtonian properties typical of concentrated non-Brownian suspensions. Indeed, the origin of shear thinning (cf. Van der Werff and De Kruif (1989)), shear 
thickening (cf. Barnes (1989); Fall et al. (2008)), yield stress (cf. Fall et al. (2009)) and normal stresses, including both the normal stress differences and the isotropic particle pressure, in non-colloidal suspensions remain unclear, as discussed in the review by Morris (2009).

Gadala-Maria (1979) was apparently the first to report normal stress differences (NSD in the following) in sheared non-Brownian suspensions, with his measurements obtained in a parallel-plate rheometer. This led to work in which Gadala-Maria and Acrivos (1980) and Kolli et al. (2002) showed irreversibility in the torque and normal stress responses, respectively, of a suspension subjected to large oscillatory straining, and Parsi and Gadala-Maria (1987) demonstrated an asymmetry in the fore-aft pair distribution function. The origin of the asymmetry in any given suspension is not clear: it may be due to the nonlinearity of multi-particle interactions, or, as asserted by Da Cunha and Hinch (1996), to contact interactions between particles that are allowed by the roughness of the particles even at low Reynolds number. The latter mechanism for asymmetric interaction is supported by the work of Rampall et al. (1997), which shows that surface roughness induces significant asymmetry in the fore and aft region of a two-particle interaction.

The issue of shear-induced normal stress has been addressed in numerical simulations, using Stokesian Dynamics (Brady and Bossis (1988)), by Sierou and Brady (2001) and in theoretical work by Brady and Morris (1997), and continuum modeling of suspension flow by Nott and Brady (1994) and Morris and Boulay (1999). The latter two studies noted here showed that particle migration may be explained as due to spatial variation of the particle NS, and can be observed at low Reynolds number (Stokes regime) but with short range forces between particles, or actual contact. Brady and Morris (1997) argued that the presence of a non-hydrodynamic interaction force or weak Brownian motion, however small, results in non-Newtonian effects such as normal stress differences. They found that the normal stresses in dilute suspension scale as $\eta \dot{\gamma} \phi^{2}$ and vanish in the purely hydrodynamic regime (when only Stokes-flow interactions are active, i.e. without Brownian motion or interparticle forces). Their work was then extended by Singh and Nott (2000) and Sierou and Brady (2002) in the hydrodynamic regime showing that normal stress differences could have hydrodynamic origin for concentrated suspensions. The prediction of Brady and Morris (1997) for the influence of the microstructural asymmetry indicates a close relation between the normal stresses and self-diffusivity of a suspension. This point is demonstrated by the experimental results of Breedveld et al. (2001, 2002) who reported anisotropy in the 
self diffusion coefficient, with differences in the velocity gradient $\left(\hat{D}_{22}\right)$ and in the vorticity direction $\left(\hat{D}_{33} \approx 0.66 \hat{D}_{22}\right)$. Attempts to deduce the complete normal stress from NSD measurements and other experimental data available for non-Brownian suspensions have been conducted by Zarraga et al. (2000) and Singh and Nott (2003). We note however that first, Zarraga et al. (2000) have used measurements obtained from the shear-induced migration of buoyant particles, the sedimentation of which may induce additional particle pressure or gradient diffusion (Martin et al. (1995)). Second, Zarraga et al. (2000) and Singh and Nott (2003) have also used the NSD of the total suspension stress, as measured through the normal force on rheometers, or through the large scale surface deformation. And these NSD of the suspension stress, may differ a priori, from the NSD of the particle phase stress (see Lhuillier (2009), for example). Quantitative measurements of the particle phase stress are not easy to perform, but they are crucially needed, as they are necessary to describe particles migration in various flow geometries (Morris and Boulay (1999)), and to address such phenomena as the shear banding instability (see for example Besseling et al. (2010)).

The first direct measurement of particle stress in a viscous regime appears to be due to Prasad and Kytömaa (1995) who measured the normal stress for fixed particle volume fraction or measured the volume fraction for an imposed normal loading in a cylindrical device that allowed the sheared suspension to suck fluid though a porous wall. The imposed normal loading approach was used by Boyer et al. (2011) to obtain very clean data relating the shear and normal stress in sheared suspensions. The results of these studies are in good general agreement with numerical evaluations of Yurkovetsky and Morris (2008), and theoretical predictions of Mills and Snabre (2009). Recently Deboeuf et al. (2009) measured the fluid pressure of a neutrally buoyant suspension in a cylindrical Couette flow and argued that fluid pressure is a good approximation of the negative of the particle pressure $\Delta \mathrm{P}^{\mathrm{f}} \approx$ $-\Pi$. The quantitative agreement of their results with previous experimental and numerical studies of Morris and Boulay (1999); Sierou and Brady (2002); Yurkovetsky and Morris (2008); Mills and Snabre (2009) supports this assertion. Interestingly, the work of Deboeuf et al. showed some dependence of the particle pressure on the size of the particles, an issue which will be addressed in the present study.

In the present paper, the experimental technique of Deboeuf et al. (2009) is described in detail and the analysis of the measurements within a rheological framework is revisited. The technique is extended to include direct measurement of individual phase and complete 
suspension normal stress at the Couette boundary. This increased capability allows measurement of the second NSD of the suspension stress. Furthermore, more accurate transducers have allowed measurement of fluid and total pressure for suspensions of volume fraction as low as $\phi=0.20$. Finally, new results show that a dependence of the measured fluid pressure with the particle size observed previously was due to particle interaction with the air-suspension interface at the top of the Couette cell.

The experimental material, set-up and method are presented in section I. Section II is devoted to the raw experimental results while section III presents interpreted results confronted with previous studies and existing models.

\section{EXPERIMENTAL SET-UP}

\section{A. Particles}

Suspensions, with particle volume fractions ranging from $\phi=0.2$ to 0.5 have been prepared with two sizes of polystyrene particles, Microbeads Dynoseeds TS 40 and TS 140, of average diameter $d_{m}^{s}=40 \mu \mathrm{m}$ and $d_{m}^{s}=140 \mu \mathrm{m}$, respectively, as reported by the vendor. The size distribution and the shape of the particles have been measured by a visual method using a Morphology G3 from Malvern Instruments. In the method, a camera mounted on an automated microscope images a layer of particles. Images are binarised, and two measurements are reported here for each particle: the average diameter and the sphericity (as noted below, this is actually determined as the circularity from simple images of the particles). From the average diameter measurements, the size distribution of each batch is constructed. Figures 1-a,b display the volume distribution of TS-40 and TS-140, respectively, with the number distribution of particles in the inset. Both distributions exhibit a well-defined peak which corresponds to the mean diameter of the distribution, $d_{m}=37 \mu \mathrm{m}$ for the TS-40 and $d_{m}=130 \mu \mathrm{m}$ for the TS 140. Figures 1-a,b show that particle distributions are far from monodisperse; for instance, there is a small fraction of particles ranging from a few microns to $200 \mu \mathrm{m}$ in the batch of TS-140. Likewise, a secondary peak at $55 \mu \mathrm{m}$ is clearly visible in the size distribution of the TS-40 (fig. 1-a). Nevertheless, $80 \%$ of the volume of TS-40 is in the interval $[34,41] \mu \mathrm{m}$, while $70 \%$ of the volume is in the range $[125,140] \mu \mathrm{m}$ for TS-

140. The average circularity $\mathcal{C}$ of the particles is classically defined by the ratio $\mathcal{C}=\frac{4 \pi \mathcal{A}}{\mathcal{P}^{2}}$ 

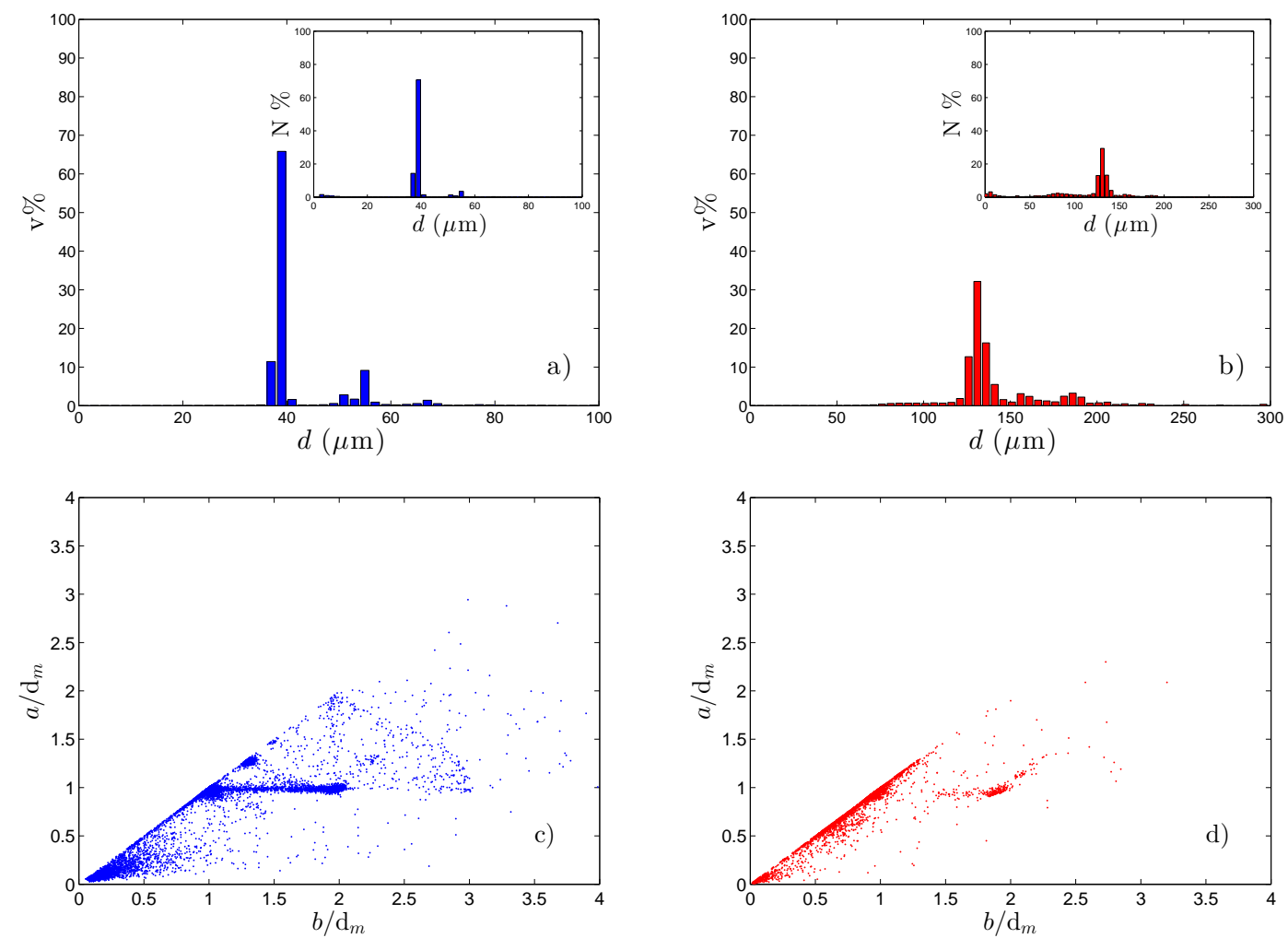

FIG. 1. Top : Size distribution of the particle used for the suspensions. a) Dynoseeds TS-40 $\left(d_{m}=37 \mu \mathrm{m}\right)$ and b) Dynoseeds TS-140 $\left(d_{m}=130 \mu \mathrm{m}\right)$. Bottom : Ellipticity of the particles. Smaller axis $a$ as a function of the larger axis $b$ normalized with the average particle diameter. c) Dynoseeds TS-40 $\left(d_{m}=37 \mu \mathrm{m}\right)$ and d) Dynoseeds TS-140 $\left(d_{m}=130 \mu \mathrm{m}\right)$. The average aspects ratios $a / b$ are equal to 0.924 for the TS-40 and to 0.927 for the TS-140.

which equals unity for a circular disk. With this definition the circularity of the TS-40 and TS-140 are very close: $\mathcal{C}_{40}=0.904$ and $\mathcal{C}_{140}=0.913$. To have a better knowledge of the noncircularity of the particles, the contour of each particle image, has been fitted with an ellipse of minor axis $a$ and major axis $b$. The average aspect ratio $a / b$ is equal to 0.924 and 0.927 for the TS-40 and TS-140 respectively. Figure 1-c,d displays the population of the particles in the plane $\left(b / d_{m}, a / d_{m}\right)$, where $a / d_{m}$ and $b / d_{m}$ represent the major and minor axis, normalized with the average diameter, $d_{m}$, of the respective distributions (i.e $d_{m}=37 \mu \mathrm{m}$ for TS-40 and $d_{m}=130 \mu \mathrm{m}$ for TS-140). On these figures spherical particles are on the line $a / d_{m}=b / d_{m}$. Figure 1 -d reveals that TS-140 are mostly spherical since only a few of them are significantly off this line. Pictures of individual particles show that the 
subset of particles of larger axis $b=2 a$ corresponds to particles in contact at the time of analysis, which reduces the number of truly non-spherical particles. TS-40 samples contain a large number of anisotropic particles (see fig.1.c). This is especially true for very small $\left(a / d_{m}<0.5\right)$ particles and particles of minor axis equal to the mean diameter $\left(a / d_{m} \approx 1\right)$. For this latter case, images of particles in this sub-set reveal that typically two particles have been stuck together during the fabrication process. Finally, AFM measurements show that the particle roughness is approximately $100 \mathrm{~nm}$, whatever the size.

\section{B. Viscosity measurements}

The carrier fluid is poly(ethylene glycol-ran-polypropylene glycol) monobutyl ether of density $\rho_{f}=1.05$ (at $20{ }^{\circ} \mathrm{C}$, with an expansion coefficient of $7.510^{-4}{ }^{\circ} \mathrm{C}^{-1}$ ) that matches the nominal density of the polystyrene particles. It has been tested, by parallel plate (of diameter $50 \mathrm{~mm}$ ) and cylindrical Couette (of diameter $10 \mathrm{~mm}$ ) rheometry (Anton Paar MCR 501), and found to be Newtonian up to a shear rate of $|\dot{\gamma}| \approx 700 \mathrm{~s}^{-1}$, with a viscosity of $\eta_{f}=2.9 \mathrm{~Pa} \cdot \mathrm{s}$ at $T=20^{\circ} \mathrm{C}$ (cf. fig. 2-a). However, the shearing of highly viscous fluids induces an increase of the fluid temperature which in turn induces a decrease of the fluid viscosity and density. To minimize this effect, fluid temperature is permanently recorded and experiments are carried out at a temperature slightly above the room temperature which allow a free cooling when the shearing is stopped. This method allow to keep temperature variations below $0.5^{\circ} \mathrm{C}$ which induces a variation of the viscosity of the order of $2 \%$ and a density variation less than $1 \%$.

The bulk effective viscosity of the suspension has been measured in parallel plate geometry where little particle migration occurs (Morris and Boulay (1999)). For each suspension, the effective viscosity has been found to be rather constant for $1 \leq|\dot{\gamma}| \leq 30 \mathrm{~s}^{-1}$. The variation of the viscosity with the solid volume fraction $\phi$ is found to be in good agreement with the empirical Krieger-Dougherty form (Krieger $(1972)): \eta_{s}=\eta_{f}\left(1-\phi / \phi_{\max }\right)^{-2}$, with $\phi_{\max }=0.625$, as shown on fig. 2 -b. The value obtained for the maximum volume fraction and the exponent of the Krieger-Dougherty law are in general agreement with values found in the literature (Stickel and Powell (2005); Ovarlez et al. (2006)). Using these suspensions, the Péclet number, the ratio of shear to Brownian motion, is $P e=6 \pi \eta_{f}|\dot{\gamma}| a^{3} / k T=O\left(10^{8}\right)$ at $|\dot{\gamma}|=1 \mathrm{~s}^{-1}$, and therefore Brownian diffusion is negligible. 

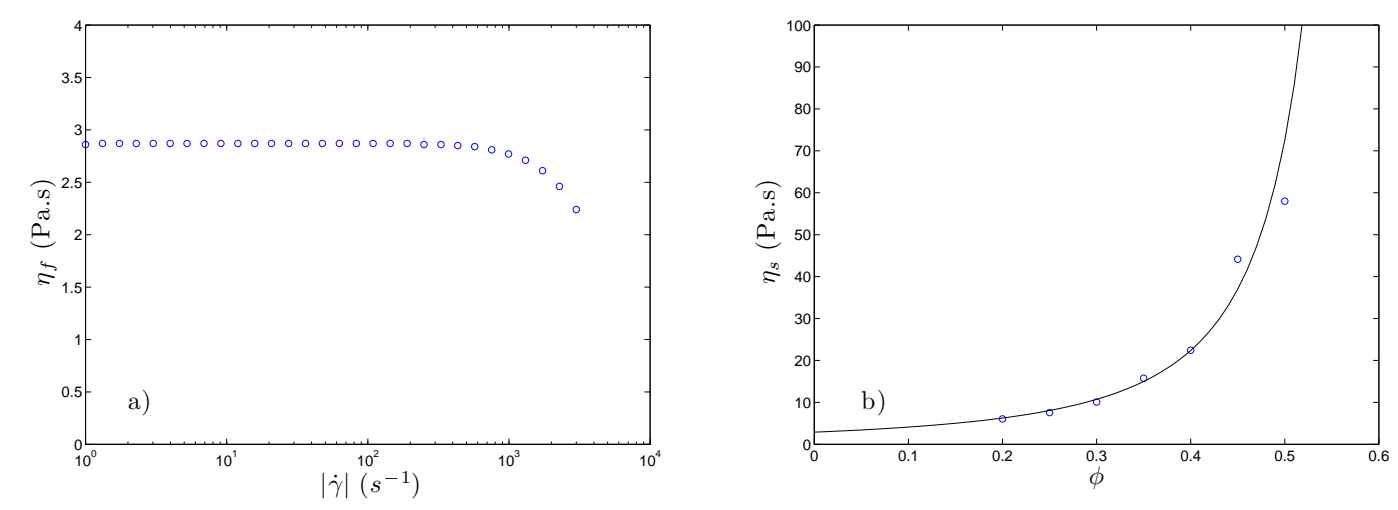

FIG. 2. Rheometry: in a $50 \mathrm{~mm}$ parallel plate geometry. (Left) Viscosity of the pure fluid as a function of the shear rate $|\dot{\gamma}|$. Measurements have been performed at $T=20^{\circ} \mathrm{C}$. (Right) Viscosity of the suspension as a function of the particle volume fraction, for $|\dot{\gamma}|=20 \mathrm{~s}^{-1}$ and $T=20^{\circ} \mathrm{C}$. The continuous line corresponds to a Krieger-Dougherty law (Krieger (1972)): $\eta_{s}=\eta_{f}\left(1-\phi / \phi_{\max }\right)^{-2}$, with $\phi_{\max }=0.625$.

\section{Experimental apparatus}

Experiments are conducted in a cylindrical Taylor-Couette cell sketched in fig. 3. The height of the cylinders is $\mathrm{h}=150 \mathrm{~mm}$. The radii of the inner and outer cylinders are, respectively, $R_{i}=17.5 \mathrm{~mm}$ and $R_{o}=20 \mathrm{~mm}$ which achieved a constant gap $b_{0}=2.5 \mathrm{~mm}$ along the axis of the cylinders. Great attention has been paid to the circularity of the two cylinders since any inhomogeneous shear will result in gradients of volume fraction, as reported by Leighton and Acrivos (1987a,b). The inner cylinder is driven by a brushless motor, while the outer cylinder is fixed. To easily fill the cell, the inner cylinder can move along the $z$ (vertical) axis.

The outer cylinder is drilled with ten holes, of diameter $6 \mathrm{~mm}$; eight holes are equipped with nylon grids of $20 \times 20 \mu \mathrm{m}^{2}$ square openings and two with latex membranes $10 \mu \mathrm{m}$ thick (cf. fig. 3). Grids and membranes are glued on small tubes of outer diameter $6 \mathrm{~mm}$, one of whose faces have been machined to match the curvature of the inner surface of the Couette cell. Each tube is filled with liquid (carrier fluid behind grids and water behind membranes) transmitting the pressure to a piezoelectric transducer (PR-23 by Keller Drukmesstechnick) of accuracy $1 \mathrm{~Pa}$. Four of the grids are placed along a circle located $20 \mathrm{~mm}$ from the bottom of the cell while the four others are along a circle $60 \mathrm{~mm}$ from the bottom of the cell. On 
each circle, grids are spaced at $90^{\circ}$ intervals. The two membranes are located $40 \mathrm{~mm}$ from the bottom of the cell and $90^{\circ}$ apart from each other, vertically aligned with two of the grids. Transducers located behind grids measure the fluid pressure in the suspension (pore pressure) while the membrane transducers measure the total pressure.
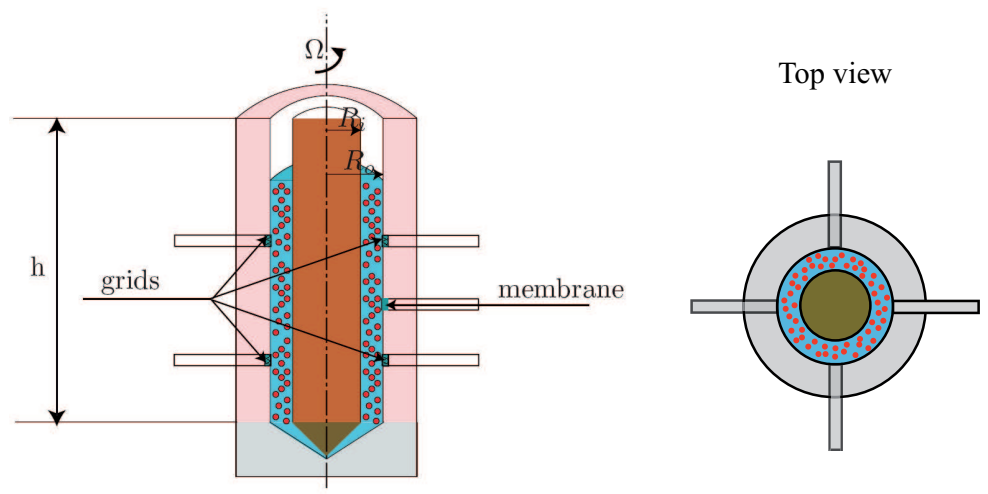

FIG. 3. Sketch of the Taylor-Couette cell.

To avoid shear induced migration to the bottom of the cell, the bottoms of the inner and outer cylinders are of conical form, so that the shear rate remains comparable in this region. Again, great attention has been paid to the inner and outer cylinder co-axiality. In a cylindrical Couette geometry, variations of the gap lead to variations of the fluid pressure. The pressure is higher upstream of a reduction of the gap and lower downstream (Acheson (1990)). The angular $(\theta)$ dependence of the pressure distribution, at a given constant rotation velocity $\Omega$, can be determined using lubrication analysis and is shown to follow the relation (Flügge (1962))

$$
\Delta P^{f}(\theta, \Omega)=P^{f}(\theta, \Omega)-P^{f}(\theta, 0)=\frac{6 \eta_{f} \Omega}{\epsilon^{2}} \frac{\lambda(2+\lambda \cos \theta) \sin \theta}{\left(2+\lambda^{2}\right)(1+\lambda \cos \theta)^{2}}
$$

where $\epsilon=b / R_{i} \ll 1, \lambda=a / b \leq 1, a$ is the eccentricity (distance between the cylinder axes), and $b(\theta)-b_{0}$ is the gap variation, maximum at $\theta=0$. The lubrication pressure with pure fluid is displayed in Figure 4, for a misalignment $a=100 \mu \mathrm{m}$ and $\dot{\gamma}=100 \mathrm{~s}^{-1}$; here the shear rate is written with its sign to indicate the importance of direction of motion. To achieve a good alignment of the cylinders, the outer cylinder is mounted on four micrometric displacement systems that allow translation in the horizontal, or $x-y$, plane and rotations around the $x$ and $y$ axes. After a rough alignment of the outer cylinder axis along the 


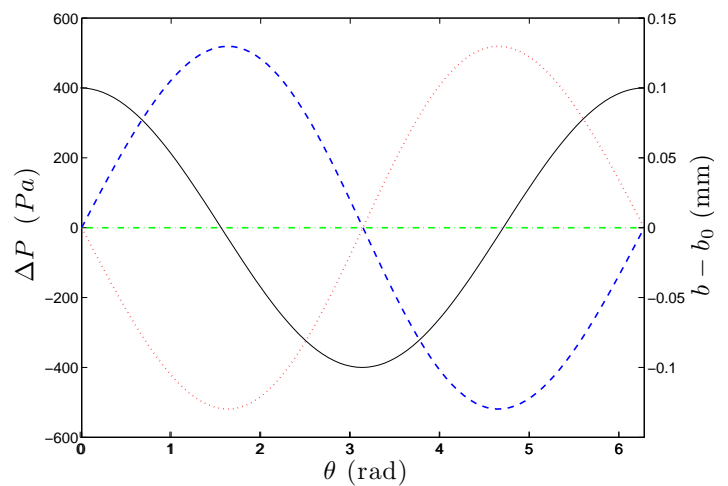

FIG. 4. Lubrication pressure, calculated in the pure liquid of viscosity $\eta_{f}=3 \mathrm{~Pa} \cdot \mathrm{s}$, for an axis misalignment $a=100 \mu \mathrm{m}$ and for $(--) \dot{\gamma}=-100 \mathrm{~s}^{-1}\left(\Omega=-14.7 \mathrm{rad} \cdot \mathrm{s}^{-1}\right),(\cdots) \dot{\gamma}=100 \mathrm{~s}^{-1},(-\cdot)$ $P^{f}(\theta, \Omega)-P^{f}(\theta,-\Omega)$ and $(-) \lambda \cos \theta$. Note the cancellation of the lubrication pressure at fixed $\theta$ when averaged over the two directions of rotation.

axis of the rotor (parallel to gravity), we use the lubrication pressures measured with the Newtonian fluid alone to align the cylinders axes: the alignment is as good as possible when the measured pressures are zero whatever the direction of rotation (c.f. Fig. 4). We note however that the replacement of the Newtonian liquid with the suspension requires moving the rotor up and down, a procedure which may result in a very slight misalignment. The
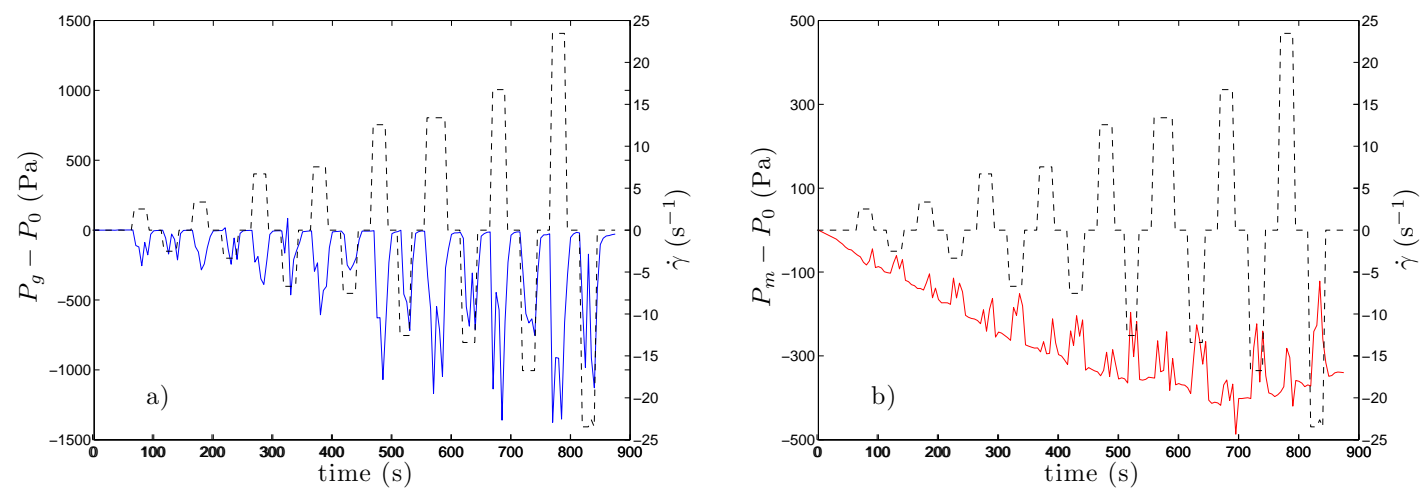

FIG. 5. (- -) Ramp of shear rate $\dot{\gamma}\left(\mathrm{s}^{-1}\right)$ (right hand axis) against time $(s)$ and (-) pressure transducer signal (left hand axis) measured a) behind one grid and b) (-) behind one impermeable membrane, for a suspension of particles of diameter $40 \mu \mathrm{m}$ at volume fraction $\phi \approx 0.5$.

importance of the residual effects of this misalignment relative to measured pressure can be 
seen in fig. 5, which displays typical pressure measurements obtained, for a step ramp of rotations, behind a grid in fig.5-a and behind a membrane in fig.5-b. As one can see, there is a systematic difference between pressure measurements realized with the inner cylinder rotating in one or the other direction. This systematic error is eliminated by averaging measurements over the two directions of rotation; this is simply because the difference in the measured pressures is a result of the lubrication pressure which is linear in the motion and thus cancels upon averaging the values for the two directions of motion.

Because the membrane pressure is transmitted through a small confined volume between the membranes and the transducers, thermal expansion induced by any temperature variation affects the pressure readings. This effect is corrected by considering only the deviation from the background pressure in the absence of shearing, $\Delta P_{m}=P_{m}^{\dot{\gamma} \neq 0}-P_{m}^{\dot{\gamma}=0}$. Figure 5-a (respectively b) shows that when a shear rate is applied to the suspension, a depression, $\Delta P_{g}$, or overpressure, $\Delta P_{m}$, is observed with an increasing magnitude of the shear rate $|\dot{\gamma}|$. To minimize shear induced migration effects (Parsi and Gadala-Maria (1987); Kolli et al. (2002); Blanc et al. (2011)), pressure measurements are performed during less than a period of rotation (one period is $14 \pi>40$ strain) and, as noted to overcome residual effects of misalignment, pressure measurements are averaged over both directions of rotation. Reversing the direction of rotation leads to transient decrease of suspension viscosity and normal stresses, which reach stationary values for a strain $|\gamma| \approx 2$ (Kolli et al. (2002) and Blanc et al. (2011)). To remove this transient effect, we discard the initial measurements after a rotation change, and average over those acquired for a strain $|\gamma|>2$ following the reversal.

Finally, measurements of grid (and membrane) pressure are averaged over the 8 (respectively 2) pressure transducers to minimize the scattering due to stress fluctuations reported in previous studies (Dasan et al. (2002); Singh et al. (2006)).

\section{EXPERIMENTS}

\section{A. Observations}

Experiments consist in measuring pressure for various shear rates. However, when the suspension is sheared above a given shear rate $\left|\dot{\gamma}_{c}\right|$, a whitish band forms at the top of our cylindrical Couette cell, as shown in fig. 6-a. The shear rate threshold depends on the 
volume fraction of the suspension and the size of the particles $\left(|\dot{\gamma}| \approx 20 \mathrm{~s}^{-1}\right.$ for TS-140 and $60 \mathrm{~s}^{-1}$ for TS-40 at $\left.\phi=0.45\right)$. Figure 6 -b displays the spatio-temporal diagram of a vertical line of fig. 6-a. The formation time is short compared to the rotation period (about half a rotation period) and the thickness of this white strip increases in time, destabilizes and becomes wavy. Once the rotation is stopped, the white strip remains. Analyzing a sample of the suspension taken in the white strip reveals that it contains almost no suspending liquid.

This "dry cream" layer has been formed by particles that have crossed the air/suspension interface. We note that corrugations of the interface had been observed by Loimer et al. (2002), Timberlake and Morris (2005) and Singh et al. (2006) in other free surface suspension flows and studied numerically by Min and Kim (2010). However, in our case the particles actually escape from the suspension. Once created, the "dry cream" thickness increases as a result of a downward air flux across its top interface (which rises), and an upward particle flux across its bottom one (which lowers). Although the bulk suspension particle fraction is not significantly decreased by the outgoing particles, the dry cream is likely to exert an axial (vertical) solid frictional stress at the wall. In such a case, our pressure measurements can no longer be directly related to the magnitude of the particle stress (see Sec. III).
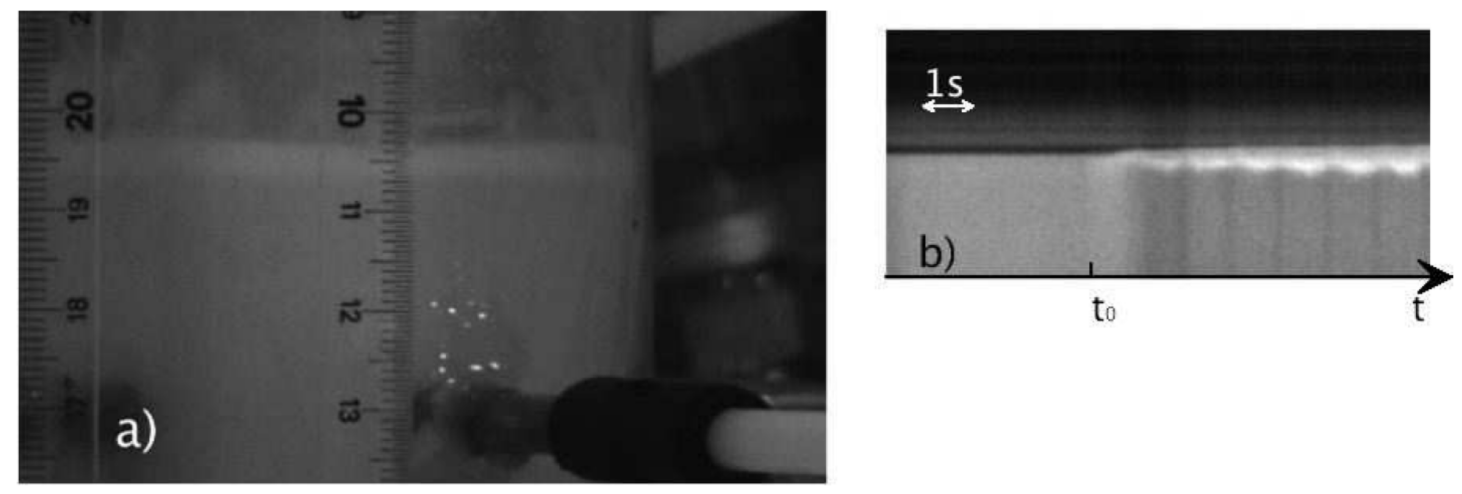

FIG. 6. a) Image of the experimental cell with a suspension of TS-140 of volume fraction $\phi=0.45$ for $|\dot{\gamma}|=45 s^{-1}$ taken 10 seconds after the beginning of the shear. b) Spatiotemporal diagram built by plotting the light intensity of a vertical line along time (horizontal direction). The white strips on the top of the suspension correspond to a "cream" of particles that have been ejected from the suspension due to shear. 


\section{B. Measurements}

The evolution of the fluid pressure (pressure behind grids) and the total stress at the outer wall (pressure behind membrane) with the shear rate have been measured for suspensions of volume fraction $\phi=0.45$ for both TS-40 and TS-140. Grid pressure, the negative of which is displayed in fig. 7-a, decreases with the absolute value of the shear rate $|\dot{\gamma}|$ until a limit value which depends on the particle size. Before this limit, grid pressure measurement is independent of particle size. Membrane pressure measurements are presented in fig 7-b. As for the grid measurements, membrane pressure increases with $|\dot{\gamma}|$ until $\left|\dot{\gamma}_{c}\right| \approx 60 \mathrm{~s}^{-1}$ beyond which measurements seem to reach a plateau, although the data are scattered. However, fig 7 exhibits a surprising particle size dependence of the total pressure. For the larger-particle suspension (using TS-140), pressure signals behind membranes are weak and measurement errors are of magnitude comparable to the measured values. However, membrane pressures seem also to reach a constant value for $|\dot{\gamma}|>20 \mathrm{~s}^{-1}$.
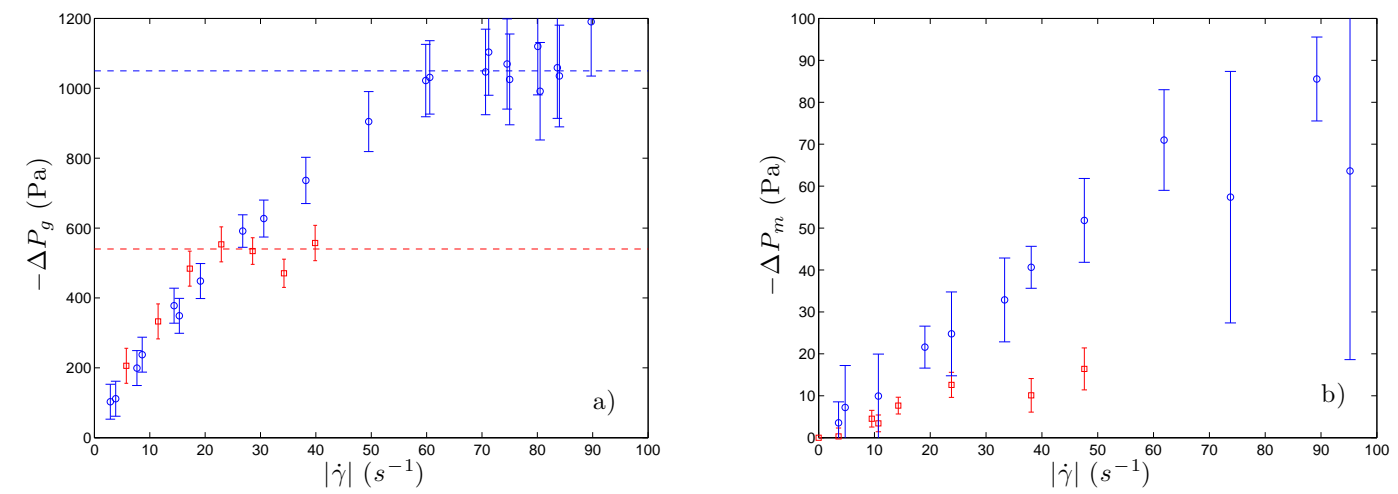

FIG. 7. Evolution of the grid (a) and membrane (b) pressure as a function of the shear rate for a suspension with volume fraction $\phi=0.45$. (o) Dynoseeds TS-40, ( $\square$ ) Dynoseeds TS-140. The two dashed lines correspond to the asymptotic values of the pressure (i.e. $540 \mathrm{~Pa}$ for TS-140 and 1050 Pa for TS-40).

The critical values $\left|\dot{\gamma}_{c}\right| \approx 60 \mathrm{~s}^{-1}$ for TS-40 and $\left|\dot{\gamma}_{c}\right| \approx 20 \mathrm{~s}^{-1}$ for TS-140 are found, beyond which pressure measurements do not evolve linearly with $|\dot{\gamma}|$. The asymptotic value depends on the particle size. This observation suggests that surface tension plays a role at the free surface. For a particle to leave the suspension the stress exerted by the surrounding mixture must be larger than the stress which keeps the particle inside, i.e. the Laplace pressure 
$\Delta P_{\mathrm{L}}=\tau / R$ where $\tau$ is the fluid-air surface tension and $R$ the curvature of the interface at the moment the particle escapes. In the experiment, the maximum pore pressure is $-\Delta P_{g_{\max }}=540$ Pa.s for TS-140 and $-\Delta P_{g_{\max }}=1050$ Pa.s for TS-40. Using the Laplace pressure to estimate an equivalent diameter, one obtains $d_{e}=\tau / \Delta P=66 \mu \mathrm{m}$ for TS-40 and $d_{e}=130 \mu \mathrm{m}$ for TS-140.

These effective diameters are in reasonable agreement with the average diameter found for the distribution of TS-140 (see Sec I A) but larger for TS-40 ( $d_{m}=37 \mu \mathrm{m}$ compared to $\left.d_{e}=66 \mu \mathrm{m}\right)$. However, the size distribution of TS-40 shows a non-negligible proportion of particles of diameter $\approx 60 \mu \mathrm{m}$ which is closer to $d_{e}$. In addition, when a particle escapes from a viscous fluid, the effective diameter is the diameter of the particle added to the thickness of the liquid film which coats each bead (Maru et al. (1971); de Gennes et al. (2004)), during the ejection process. This leads to an effective diameter larger than the particle, and could explain the remaining discrepancy between the estimated Laplace pressure and the asymptotic one measures for the suspension of TS-40. These measurements support the interpretation of the formation of the cream at the top of the suspension, and provide a likely explanation for the size dependence of particle pressure reported by Deboeuf et al. (2009). Consequently, all further results are for experiments conducted for shear rates below $\left|\dot{\gamma}_{c}\right|$ for each suspension. Note that the grid and membrane pressures evolve linearly with the shear rate for $|\dot{\gamma}| \leq 20 \mathrm{~s}^{-1}$ for both TS-40 and TS-140 so that in this range $\Delta P_{g} /|\dot{\gamma}|$ and $\Delta P_{m} /|\dot{\gamma}|$ depend only on the volume fraction $\phi$.

Variations of grid and membrane pressure with $\phi$ are presented, with normalization by $\eta_{f}|\dot{\gamma}|$. Evolution of $\Delta P_{g} / \eta_{f}|\dot{\gamma}|$ and $\Delta P_{m} / \eta_{f}|\dot{\gamma}|$ are displayed on fig. 8 for the TS-40 and TS140 suspensions. The dependence of $\Delta P_{g}$ and $\Delta P_{m}$ on $\phi$ is clearly nonlinear, and we note that $\Delta P_{g}$ is independent of the particle size. The insets of fig. 8-a and b present respectively the evolution of the grid and membrane pressures, normalized with the suspension shear stress in logarithmic scale. We note that the shear stress is not measured in our device, but is estimated by the product of the imposed shear rate with the viscosity law (Ovarlez et al. (2006)) obtained in a parallel plate device (see fig. 2-b). 

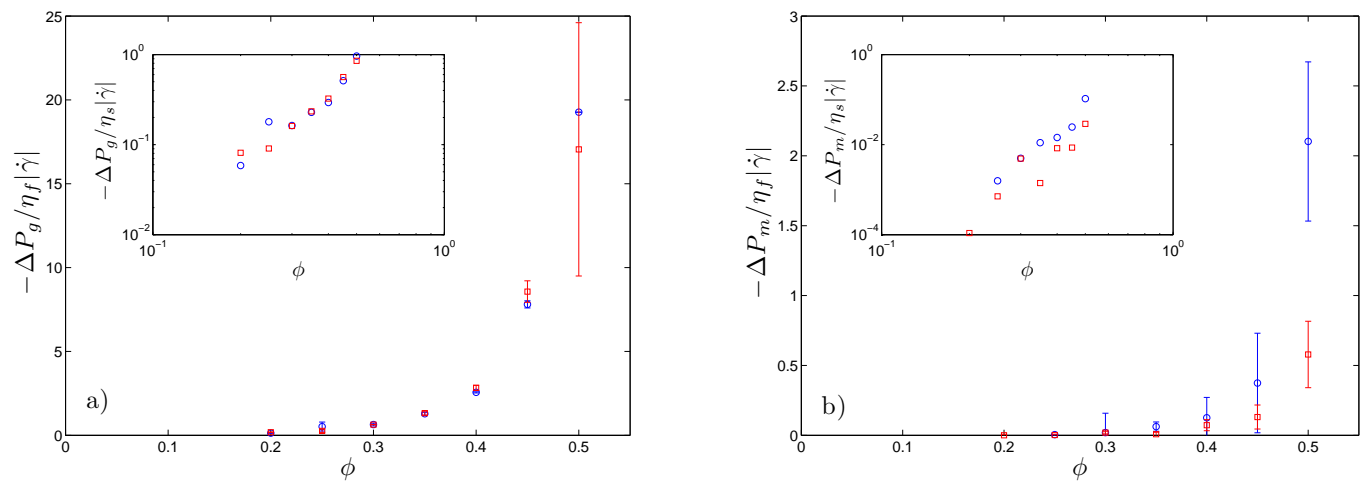

FIG. 8. Evolution of the dimensionless grid (a) and membrane (b) pressure as a function of the particle volume fraction. Pressures are normalised by $\eta_{f}|\dot{\gamma}|$. (०) Dynoseeds TS-40 and ( $\square$ ) Dynoseeds TS-140. The insets display in logarithmic scale the pressures normalized with the shear stress of the suspension $\left(\eta_{s}|\dot{\gamma}|\right)$.

\section{RESULTS AND DISCUSSION}

\section{A. Normal Stresses}

To relate the measurements to suspension rheology, grid and membrane pressure must be translated into normal stress components. In the absence of inertia, the momentum equations, for the two phases, particles and fluid (Jackson (1997); Nott and Brady (1994); Morris and Boulay (1999); Lhuillier (2009)), are derived from the Cauchy equations:

$$
\begin{gathered}
\nabla \cdot \Sigma^{p}+\boldsymbol{F}^{\boldsymbol{H}}+\Phi\left(\rho_{p}-\rho_{f}\right) \boldsymbol{g}=\mathbf{0} \\
\nabla \cdot \boldsymbol{\Sigma}^{f}-\boldsymbol{F}^{\boldsymbol{H}}+\rho_{f} \boldsymbol{g}=\mathbf{0}
\end{gathered}
$$

where $\boldsymbol{F}^{\boldsymbol{H}}$ is the friction force, proportional to the difference between the phase averaged velocities, $\left\langle\boldsymbol{v}>^{p}-\left\langle\boldsymbol{v}>^{f}, \boldsymbol{\Sigma}^{f}\right.\right.$ is the fluid phase stress, and $\boldsymbol{\Sigma}^{p}$ is the particle phase stress, in which the Archimedes force has been included for convenience (Lhuillier (2009)). In our experiment, the suspension is neutrally buoyant, $\rho_{p}=\rho_{f}=\rho$, and (2) reduces to

$$
\nabla \cdot \Sigma^{p}+F^{H}=0 .
$$

We note that a stress balance equation for the suspension is obtained by the sum of (3) and (2):

$$
\nabla \cdot \boldsymbol{\Sigma}+\rho \boldsymbol{g}=\mathbf{0},
$$


where the total stress of the suspension,

$$
\Sigma=\Sigma^{p}+\Sigma^{f}
$$

is also related to the phase averaged stresses $\boldsymbol{\Sigma}=(1-\Phi)<\boldsymbol{\sigma}>^{f}+\Phi<\boldsymbol{\sigma}>^{p}$ (Jackson (1997)), although $\Sigma^{f} \neq(1-\Phi)<\boldsymbol{\sigma}>^{f}$ and $\boldsymbol{\Sigma}^{p} \neq \Phi<\boldsymbol{\sigma}>^{p}$ a priori (Lhuillier (2009); Nott et al. (2011)). Note that the description of suspension flows, using $\boldsymbol{\Sigma}$, requires nevertheless the concentration field of the particles, which results from the migration described by either (3) or (4). As a consequence, unlike the normal stress differences of $\boldsymbol{\Sigma}$ which may be neglected in most suspension flows, the normal stress components of $\Sigma^{f}$ or $\Sigma^{p}$, are definitely needed to describe the dynamics of the migration of the particles, or at least the stationary (or quasistatic) migrated state, obtained for $\langle\boldsymbol{v}\rangle^{p}-\langle\boldsymbol{v}\rangle^{f}=0$, which corresponds to $\nabla \cdot \boldsymbol{\Sigma}^{\boldsymbol{P}}=0$ as shown by (4); for the case of present interest, this means a uniform particle phase normal stress in the cross-stream direction. The originality of our experiment is that it addresses a situation where no migration occurs, but which nevertheless provides a measurement of the phase stresses in the radial direction, as will be shown in the following.

We note first that to relate our pressure measurements to rheological properties, one should remove the hydrostatic pressure. Assuming that the shearing of the suspension does not generate any tangential stress in the vorticity direction, (which is true if there is no solid friction along the $z$-axis of the walls, i.e. no dry cream layer),

$$
\Sigma_{z r}=\Sigma_{z \theta}=0
$$

and the $z$-projection of (5) is written $\frac{\partial \Sigma_{z z}}{\partial z}+\rho g=0$, and can be solved, with the condition that the pressure at the free surface, $z=h_{s}$ is the atmospheric pressure, $P_{a}$ :

$$
\Sigma_{z z}=-P_{a}+\rho g\left(z-h_{s}\right) .
$$

As expected, when the suspension is at rest $(\dot{\gamma}=0)$, the weight of the suspension is balanced by $\sum_{z z}^{\dot{\gamma}=0}$, which obeys a hydrostatic equation.

Moreover, when the suspension is sheared, $h_{s}$ remains uniform in our experiments: the centrifugal forces, the lubrication pressures, and the anti-Weissenberg effect (see for instance Zarraga et al. (2000)) do not generate any significant large scale (much larger than the 
particles diameter a) deformation of the interface. As a result, the right hand side of (8) remains unchanged, and the variation due to the shearing, $\Delta \Sigma_{z z}=\Sigma_{z z}-\Sigma^{\dot{\gamma}=0}$ is null:

$$
\Delta \Sigma_{z z}=0
$$

In other words, the hydrostatic contribution (i.e. the $z$-varying offset of $\Sigma_{i i}, \Sigma_{i i}^{f}, \Delta P_{m}$ and $\left.\Delta P_{g}\right)$ remains unchanged when the shear is applied. To remove this hydrostatic $z$ dependence, we introduce the variations $\Delta N=N^{\dot{\gamma} \neq 0}-N^{\dot{\gamma}=0}$, of a generic normal stress $N$, induced by shearing at $|\dot{\gamma}| \neq 0$. Due to the cylindrical geometry of the setup, $\Delta N$ does not depend on $\theta$ either. As a consequence, all the variations $\Delta N$, measured or evaluated at $r=R_{o}$, can be combined or compared. They represent actual shear-induced normal stresses, and are thus expected to vary linearly with $|\dot{\gamma}|$ in the Stokesian regime studied here.

In our setup, there is no radial flux of either the fluid or particle phase across the grids. On one hand, the particles are stopped by the grids, which means that the radial particle phase stress is balanced by the grids. On the other hand, the radial fluid phase stress (inside the Couette device) must be balanced by the "grid pressure", $P_{g}=-\Sigma_{r r}^{f}$, which we write in terms of variations:

$$
\Delta P_{g}=-\Delta \Sigma_{r r}^{f}
$$

The balance between normal stresses (and their variations) on both sides of the membrane is written, with the use of (6):

$$
\Delta P_{m}=-\Delta \Sigma_{r r}=-\Delta \Sigma_{r r}^{p}-\Delta \Sigma_{r r}^{f}
$$

From a combination of (11) and (10), one gets the radial particle stress:

$$
\Delta \Sigma_{r r}^{p}=\Delta P_{g}-\Delta P_{m}
$$

Moreover, the second normal stress difference of the suspension stress, $N_{2}=\Sigma_{22}-\Sigma_{33}=$ $\Sigma_{r r}-\Sigma_{z z}(2$ and 3 being the directions of the velocity gradient and the vorticity of the bulk flow, respectively), is obtained by adding (11) and (9):

$$
N_{2}=\Delta \Sigma_{r r}-\Delta \Sigma_{z z}=-\Delta P_{m}
$$


To summarize, the membrane pressure, $\Delta P_{m}$, the grid pressure, $\Delta P_{g}$, and their combination, $\left(\Delta P_{g}-\Delta P_{m}\right)$, provide, respectively, measurements of the second normal stress difference, $N_{2}$, as shown by (13) of the total stress of the suspension, $\boldsymbol{\Sigma}$; of the second (i.e. radial) normal stress component of the fluid phase stress, $\Sigma^{f}$, as shown by (10); and of the particle phase stress, $\Sigma^{p}(12)$, in a cylindrical Couette geometry.

Figure 9 displays the normalized second normal stress difference $\left(-N_{2}=\Delta P_{m}\right)$, measured for the suspensions containing the beads TS-40 and TS-140, as a function of the volume fraction $\phi$ in a semilog scale. For the sake of comparison, numerical predictions of Sierou and Brady (2002), using Stokesian Dynamics, and of Yeo and Maxey (2010), based on Force Coupling Method, are also displayed in fig. 9, together with experimental results of Zarraga et al. (2000), Singh and Nott (2003) and Couturier et al. (2011).

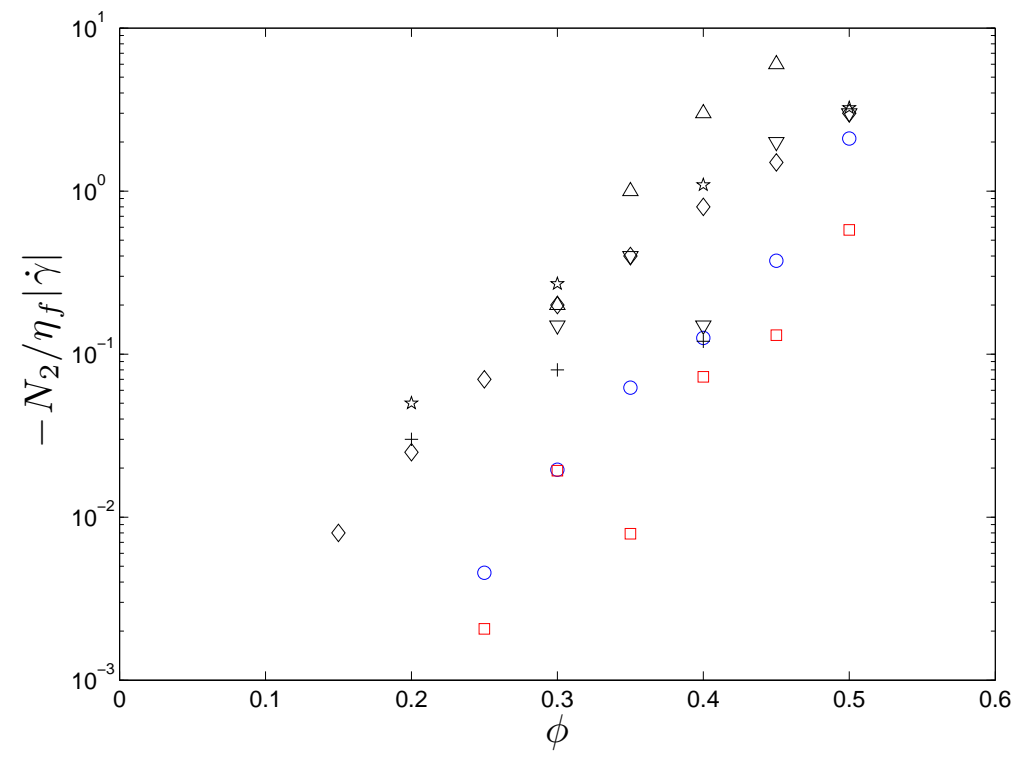

FIG. 9. Variation of the normalized (relative to the shear stress of the pure liquid) second normal suspension stress difference $N_{2}$, with the volume fraction $\phi$. (o) Dynoseeds TS-40, ( $\square$ Dynoseeds

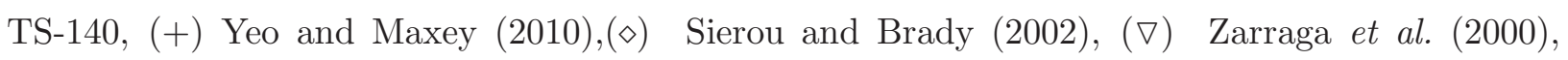
$(\triangle)$ Singh and Nott (2003) and $(\star)$ Couturier et al. (2011).

Our two sets of data exhibit a linear behavior on this semilog plot, supporting an exponential increase of the form $\exp (20 \phi)$. Such behavior is also observed for the data reported by Singh and Nott (2003) in the same geometry, but with a significantly higher pre-factor in 
that work. We have checked the magnitude of $N_{2}$ by carrying out an experiment, at $\phi=0.45$, with capillary tubes directly connected to the suspension (with no grid and no membrane): when the suspension was sheared, the liquid level increased only by a few millimeters, in line with the membrane pressures obtained for the same shear rates. The discrepancy between our results and other measurements remains to be explained and although our low $N_{2}$ values could be due to the small roughnesses of our beads (Davis et al. (2003)), it is likely that other explanations come into play. One of the reasons that may explain the difference with measurements of Singh and Nott (2003), performed in the same geometry, could be the lubrication effect discussed in paragraph IC, which to our knowledge has not been canceled in the Singh and Nott (2003) experiment. Moreover, in contrast with the bulk estimations of simulations, we actually perform measurements at a wall, which may modify the hydrodynamic interactions between the particles (Zurita-Gotor et al. (2007)), but may also play a role through its roughness. There also exists a kind of boundary layer at the wall, in which the particle concentration decreases from its bulk value to zero. The description of this boundary layer (in term of boundary conditions) is not well-developed, and its influence on our measurements is not clear. Moreover, the particle stress tensor, $\boldsymbol{\Sigma}^{p}$, has been assumed to depend on the local shear alone, and not on the velocity field curvature. However, it is well known that the trajectory of a single particle is affected by any flow curvature (Faxén law), and it is likely that the flow curvature (i.e. the geometry of the device, cylindrical, plate-plate, cone-plate ...) also some influence on the particle stress. This could explain in part the difference between our $N_{2}$ direct measurements, at a wall of a cylindrical geometry, and those by Couturier et al. (2011), at the free surface of a tilted trough setup: It may be due to the differences in the geometry and in the nature of the boundary conditions (i.e. free or solid surface).

The particle phase radial normal stress, $\Delta \Sigma_{r r}^{p}=\Delta P_{g}-\Delta P_{m}$, normalized with the viscous shear stress of the suspension, $\left|\Sigma_{12}\right|=\eta_{s}|\dot{\gamma}|$, is plotted as a function of $\phi$ and $\phi^{2}$, in fig. 10-a and $10-\mathrm{b}$, respectively.

Previous experiments (e.g. Zarraga et al. (2000); Singh and Nott (2003); Couturier et al. (2011)) reported normal stress differences obtained from classical rheometry or interface deformation and then related to the suspension stress $\boldsymbol{\Sigma}$ which could differ from the normal stress difference of the particle phase (Lhuillier, 2009). Nonetheless, we compare our measurements to those reported by Zarraga et al. (2000) and our data compare fairly well 

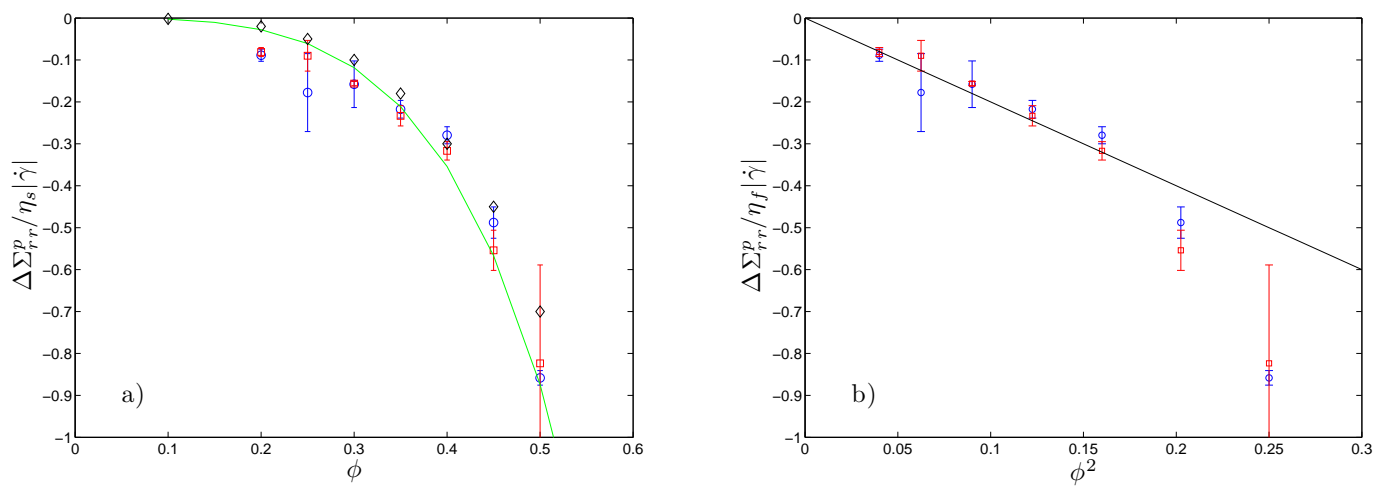

FIG. 10. $\Delta \Sigma_{r r}^{p}$, normalized with the suspension shear stress, as a function of $\phi$ (a) and of $\phi^{2}$ (b). Our data, (०) Dynoseeds TS-40 and ( $\square$ ) Dynoseeds TS-140, are compared to the data $(\diamond)$ of Sierou and Brady (2002) and the fit (solid line) of Zarraga et al. (2000) in (a), and to $y=-2 \phi^{2}$ (solid straight line) in (b).

with the fit, $\Sigma_{r r}^{p}=-2.17 \phi^{3} \exp (2.34 \phi) \eta_{s} \dot{\gamma}$, obtained by Zarraga et al. (2000) (solid line in fig. $10(\mathrm{a}))$. However, our measurements are slightly larger in absolute value, which is in accordance with the asymptotic behavior, $\Sigma_{r r}^{p}=-2 \phi^{2} \eta_{s}|\dot{\gamma}|$, obtained at low enough particle volume fraction, $\phi<0.4$, in fig. 10 (b). This quadratic dependence of $\Sigma_{r r}^{p} /\left(\eta_{s}|\dot{\gamma}|\right)$ agrees with the theoretical particle pressure prediction of Brady and Morris (1997) for small $\phi$, and supports the contention that the particle phase normal stress $\Sigma_{r r}^{p}$ is due to asymmetric pair interactions under dilute conditions, and may not require many-body effects. We note that our measurements of $\Sigma_{r r}^{p}$ displayed on fig. 10 (b) have been obtained for particle concentrations as low as $\phi=0.2$, and do not appear to suggest a concentration threshold. The data deviation from the $\phi^{2}$ scaling for $\phi>0.4\left(\phi^{2}>0.16\right)$.

For the sake of completeness, we compare in Fig. 11 our measured values, $\Sigma_{r r}^{p} /\left(\eta_{f}|\dot{\gamma}|\right)$, with the recent expression proposed by Prof. P. Mills (private communication) and by Boyer et al. (2011) for the particle pressure $\Pi /\left(\eta_{f}|\dot{\gamma}|\right)=\left(\phi_{\max } / \phi-1\right)^{-2}$, with $\phi_{\max }=0.605$, based on the determination of relaxation time $\tau_{r}=\eta_{s} / P^{s}$ where $P^{s}$ is the particle pressure used to define the viscous analog of the "inertial number" introduced by da Cruz et al. (2002) for granular media. Although $\Sigma_{r r}^{p} /\left(\eta_{f}|\dot{\gamma}|\right)$ actually represents only one component of the normal particle phase stress, it compares remarkably well with the simple analytical expression for the particle pressure. The normalization (by $\eta_{f}|\dot{\gamma}|$ ) used in fig. 11 enables 


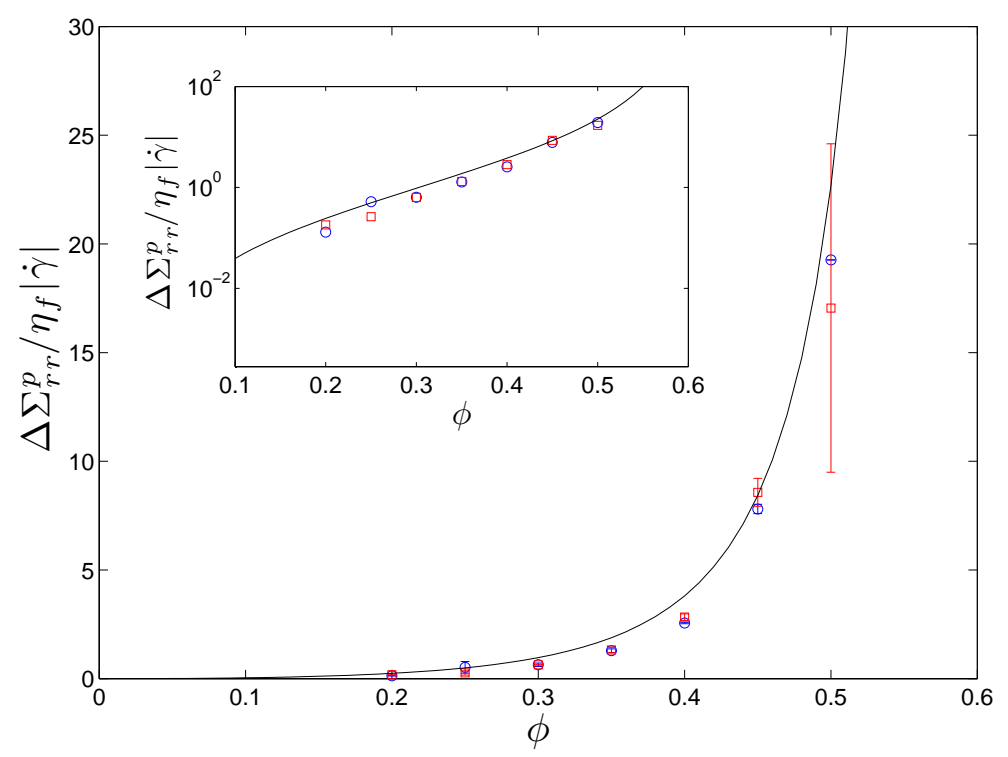

FIG. 11. Comparison of $-\Sigma_{r r}^{p}$, normalized with the fluid shear stress, $\eta_{f}|\dot{\gamma}|$, with the theoretical prediction for the particle pressure, $\Pi / \eta_{f}|\dot{\gamma}|$, proposed by Mills (2011).

the comparison with $N_{2}$ displayed in fig 9. $\Sigma_{r r}^{p}$ exceeds the fluid shear stress $\eta_{f}|\dot{\gamma}|$, for $\phi \gtrsim \phi_{\max } / 2\left(\phi_{\max } \approx 0.6\right)$ (whereas, with the exception of one measurement at $\phi=0.5$, the values obtained for $N_{2}$ were always below $\eta_{f}|\dot{\gamma}|$. More generally, we recall that our pressure measurements always obeyed $\left|\Delta P_{g}\right| \gg\left|\Delta P_{m}\right|$ (fig. 8) which implies, from (13), (12) and $(10)$,

$$
\left|N_{2}\right| \ll\left|\sum_{r r}^{p}\right|
$$

and

$$
\Delta \Sigma_{r r}^{p} \approx \Delta P_{g}=-\Delta \Sigma_{r r}^{f}
$$

Thus, the grid pressure provides an approximate measurement of the particle phase radial normal stress, $\Delta \Sigma_{r r}^{p} \approx \Delta P_{g}$, and a good estimate of the particle pressure if one assumes that the normal stress difference of $\Sigma^{p}$ is of substantially smaller magnitude. The normal stress difference, $N_{2}$, of the suspension stress, $\Sigma$, is found to be small when compared to the particle phase normal stress $\Sigma_{r r}^{p}$. 


\section{CONCLUSION}

The method for measuring a normal stress component of the particle phase in a Stokesian suspension through measurements of the grid pressure variation $\Delta P_{g}$ induced by shear in a Taylor-Couette device (Deboeuf et al. (2009)) has been revisited, including a measurement of the total suspension radial stress $\left(\Sigma_{r r}\right)$ obtained from the pressure variation behind an impermeable membrane $\Delta P_{m}$. Analysis shows that the latter is indeed needed to provide access to the particle phase radial normal stress $\Sigma_{r r}^{p}=\Delta P_{g}-\Delta P_{m}$. Moreover, we showed that $\Delta P_{m}$ is also a measurement of the second normal stress difference $N_{2}$, of the total stress of the suspension, $\boldsymbol{\Sigma}$. Measurements of $N_{2}$ by the method described in this work exhibit a linear dependence with the shear rate $|\dot{\gamma}|$ and an exponential variation with the particle volume fraction $\phi$, in line with the results of previous studies (Sierou and Brady (2002), Yeo and Maxey (2010), Zarraga et al. (2000) and Couturier et al. (2011)); they are also found to be one order of magnitude lower than our measured values of $\Sigma_{r r}^{p}$, which can be estimated, therefore, by the grid pressure alone, $\Sigma_{r r}^{p} \simeq \Delta P_{g}$.

Our measurements of $\Sigma_{r r}^{p}$ show no particle size dependence and increase linearly with the shear rate $|\dot{\gamma}|$. When normalized by the suspension shear stress, $\eta_{s}|\dot{\gamma}|$, they increase quadratically with $\phi$, in the range $0<\phi<0.4$, which agrees with the theoretical particle pressure prediction of Brady and Morris (1997) for small $\phi$, and supports the contention that the particle phase normal stress $\Sigma_{r r}^{p}$ is due to asymmetric pair interactions under dilute conditions, and may not require many-body effects. When normalized by the fluid shear stress, $\eta_{f}|\dot{\gamma}|$, these measurements are found to be nicely described by the analytic expression for the particle pressure $\Pi /\left(\eta_{f}|\dot{\gamma}|\right)=\left(\phi_{\max } / \phi-1\right)^{-2}$, recently used to describe experimental data by Boyer et al. (2011) and for high $\phi$ having the same functional form as the constitutive relation proposed by Morris and Boulay (1999).

The authors would like to thank GdR MEPHY and MEGE, Dr. A. Deboeuf, Dr. E. Lemaire, Dr. D. Lhuilier, Prof. P. Mills, and Prof. D. Salin for fruitful discussions, as well as A. Aubertin and R. Pidoux for the design and the realization of the experimental set-up and acquisition procedure. This work was partly supported by the Triangle de la Physique, ANR Coliner and NSF PREM (DMR-0934206) "Dynamics of Heterogeneous and Particulate Materials." 


\section{REFERENCES}

Acheson D., Elementary fluid dynamics (Clarendon Press, 1990).

Barnes H. A., "Shear-thickening ("dilatancy") in suspensions of nonaggregating solid particles dispersed in Newtonian liquids," J Rheol., 33, 329-366 (1989).

Batchelor G. K. and Green J. T., "The determination of the bulk stress in a suspension of spherical particles to order c ${ }^{2}, "$ J. Fluid Mech., 56, 401-427 (1972).

Besseling R., Isa L., Ballesta P., Petekidis G., Cates M. E., and Poon W. C. K., "Shear banding and flow-concentration coupling in colloidal glasses," Phys. Rev. Lett., 105, 268301 (2010).

Blanc F., Peters F., and Lemaire E., "Local transient rheological behavior of concentrated suspensions," J. Rheol., 55, 835-854 (2011).

Bonnoit C., Lanuza J., Lindner A., and Clement E., "Mesoscopic length scale controls the rheology of dense suspensions," Phys. Rev. Lett., 105, 108302 (2010).

Boyer F., Guazzelli E., and Pouliquen O., "Unifying suspension and granular rheology," Phys. Rev. Lett., 107, 188301 (2011).

Brady J. F. and Bossis G., "Stokesian dynamics," Annual Review of Fluid Mechanics, 20, 111-157 (1988).

Brady J. F. and Morris J. F., "Microstructure of strongly sheared suspensions and its impact on rheology and diffusion," J. Fluid Mech., 348, 103-139 (1997).

Breedveld V., van den Ende D., Bosscher M., Jongschaap R. J. J., and Mellema J., "Measuring shear-induced self-diffusion in a counterrotating geometry," Phys. Rev. E, 63, 021403 (2001).

Breedveld V., van den Ende D., Bosscher M., Jongschaap R. J. J., and Mellema J., "Measurement of the full shear-induced self-diffusion tensor of noncolloidal suspensions," The Journal of Chemical Physics, 116, 10529-10535 (2002).

Chow A. W., Sinton S. W., Iwamiya J. H., and Stephens T. S., "Shear-induced particle migration in Couette and parallel-plate viscometers: NMR imaging and stress measurements," Physics of Fluids, 6, 2561-2576 (1994).

Couturier E., Boyer F., Pouliquen O., and Guazzelli E., "Suspensions in a tilted trough: second normal stress difference," J. Fluid Mech., 686, 26-39 (2011).

da Cruz F., Chevoir F., Bonn D., and Coussot P., "Viscosity bifurcation in granular mate- 
rials, foams, and emulsions," Phys. Rev.E, 66, 051305 (2002).

Da Cunha F. R. and Hinch E. J., "Shear-induced dispersion in a dilute suspension of rough spheres." J. Fluid Mech., 309, 211-223 (1996).

Dasan J., Ramamohan T. R., Singh A., and Nott P. R., "Stress fluctuations in sheared Stokesian suspensions," Phys. Rev. E, 66, 021409 (2002).

Davis R. H., Zhao Y., Galvin K. P., and Wilson H. J., "Solid-solid contacts due to surface roughness and their effects on suspension behaviour," Phil. Trans. R. Soc. Lond. A 15, 361, 871-894 (2003).

Deboeuf A., Gauthier G., Martin J., Yurkovetsky Y., and Morris J. F., "Particle pressure in a sheared suspension: A bridge from osmosis to granular dilatancy," Phys. Rev. Lett., 102, 108301 (2009).

Einstein A., "Über die von der molekularkinetschen theorie der wärme geforderte bewegung in ruhenden flüssigeiten suspendierten teilchen," Annalen der Physik, 17, 549-560 (1905).

Fall A., Bertrand F., Ovarlez G., and Bonn D., "Yield stress and shear banding in granular suspensions," Phys. Rev. Lett., 103, 178301 (2009).

Fall A., Huang N., Bertrand F., Ovarlez G., and Bonn D., "Shear thickening of cornstarch suspensions as a reentrant jamming transition," Phys. Rev. Lett., 100, 018301 (2008).

Flügge W., Handbook of Engineering Mechanics, 1st ed., edited by W. Flügge (Mc Graw Hill, 1962).

Gadala-Maria F., The Rheology of Concentrated Suspensions, Ph.D. thesis, Stanford University (1979).

Gadala-Maria F. and Acrivos A., "Shear-induced structure in a concentrated suspension of solid spheres," J. Rheol., 24, 799-814 (1980).

Gauthier G., Martin J., and Salin D., "Gravity waves at the interface between miscible fluids and at the top of a settling suspension," Phys. Rev. Lett., 94, 204501 (2005).

de Gennes P.-G., Brochard-Wyart F., and Quere D., Capillarity and Wetting Phenomena: Drops, Bubbles, Pearls, Waves (Springer Berlin / Heidelberg, 2004).

Jackson R., "Locally averaged equations of motion for a mixture of identical spherical particles and a Newtonian fluid," Chemical Engineering Science, 52, 2457-2469 (1997).

Kolli V., Pollauf E. J., and Gadala-Maria F., "Transient normal stress response in a concentrated suspension of spherical particles," J. Rheol., 46, 321-334 (2002).

Krieger I., "Rheology of monodisperse latices," Adv. Colloids Interface Sci., 3, 111 (1972). 
Leighton D. and Acrivos A., "Measurement of shear-induced self-diffusion in concentrated suspensions of spheres," J. Fluid Mech., 177, 109-131 (1987a).

Leighton D. and Acrivos A., "The shear-induced migration of particles in concentrated suspensions," J. Fluid Mech., 181, 415-439 (1987b).

Lhuillier D., "Migration of rigid particles in non-Brownian viscous suspensions," Physics of Fluids, 21, 1-6 (2009).

Loimer T., Nir A., and Semiat R., "Shear-induced corrugation of free interfaces in concentrated suspensions," Journal of Non-Newtonian Fluid Mechanics, 102, 115 - 134 (2002).

Martin J., Rakotomalala N., and Salin D., "Hydrodynamic dispersion of noncolloidal suspensions: Measurement from Einstein's argument," Phys. Rev. Lett., 74, 1347-1350 (1995).

Maru H., Wasan D., and Kintner R., "Behavior of a rigid sphere at a liquid-liquid interface," Chemical Engineering Science, 26, 1615 - 1628 (1971).

Mills P., "private communication," (2011).

Mills P. and Snabre P., "Apparent viscosity and particle pressure of a concentrated suspension of non-Brownian hard spheres near the jamming transition," The European Physical Journal E: Soft Matter and Biological Physics, 30, 309-316 (2009).

Min K. H. and Kim C., "Simulation of particle migration in free-surface flows." AIChE Journal, 56, 2539-2550 (2010).

Morris J. F., "A review of microstructure in concentrated suspensions and its implications for rheology and bulk flow," Rheologica Acta, 48, 909-923 (2009).

Morris J. F. and Boulay F., "Curvilinear flows of noncolloidal suspensions: The role of normal stresses," J. Rheol., 43, 1213-1237 (1999).

Nott P., Guazzelli E., and Pouliquen O., "The suspension balance model revisited," Physics of Fluids, 23, 043304 (2011).

Nott P. R. and Brady J. F., "Pressure-driven flow of suspensions: simulation and theory," J. Fluid Mech., 275, 157-199 (1994).

Ovarlez G., Bertrand F., and Rodts S., "Local determination of the constitutive law of a dense suspension of noncolloidal particles through Magnetic Resonance Imaging," J. Rheol., 50, 259-292 (2006).

Parsi F. and Gadala-Maria F., "Fore-and-aft asymmetry in a concentrated suspension of solid spheres." J. Rheol., 31, 725-732 (1987).

Phillips R. J., Armstrong R. C., Brown R. A., Graham A. L., and Abbott J. R., “A 
constitutive equation for concentrated suspensions that accounts for shear-induced particle migration," Physics of Fluids A: Fluid Dynamics, 4, 30-40 (1992).

Prasad D. and Kytömaa H. K., "Particle stress and viscous compaction during shear of dense suspensions," International Journal of Multiphase Flow, 21, 775 - 785 (1995).

Rampall I., Smart J. R., and Leighton D. T., "The influence of surface roughness on the particle-pair distribution function of dilute suspensions of non-colloidal spheres in simple shear flow." J. Fluid Mech., 339, 1-24 (1997).

Sierou A. and Brady J. F., "Accelerated Stokesian dynamics simulations," J. Fluid Mech., 448, 115-146 (2001).

Sierou A. and Brady J. F., "Rheology and microstructure in concentrated noncolloidal suspensions," J. Rheol., 46, 1031-1056 (2002).

Singh A., Nir A., and Semiat R., "Free-surface flow of concentrated suspensions," International Journal of Multiphase Flow, 32, 775 - 790 (2006).

Singh A. and Nott P. R., "Normal stresses and microstructure in bounded sheared suspensions via Stokesian dynamics simulations.” J. Fluid Mech., 412, 279-301 (2000).

Singh A. and Nott P. R., "Experimental measurements of the normal stresses in sheared Stokesian suspensions," J. Fluid Mech., 490, 293-320 (2003).

Stickel J. J. and Powell R. L., "Fluid mechanics and rheology of dense suspensions." Annual Review of Fluid Mechanics, 37, 129-149 (2005).

Timberlake B. and Morris J. F., "Particle migration and free-surface topography in inclined plane flow of a suspension," J. Fluid Mech., 538 (2005).

Van der Werff J. C. and De Kruif C. G., "Hard-sphere colloidal dispersions: The scaling of rheological properties with particle size, volume fraction, and shear rate," J. Rheol., 33, 421-454 (1989).

Yeo K. and Maxey M. R., "Dynamics of concentrated suspensions of non-colloidal particles in Couette flow." J. Fluid Mech., 649, 205-231 (2010).

Yurkovetsky Y. and Morris J. F., "Particle pressure in a sheared Brownian suspension," J Rheol, 52, 141 (2008).

Zarraga I. E., Hill D., and Leighton D., "The characterization of the total stress of concentrated suspensions of noncolloidal spheres in Newtonian fluids," J. Rheol., 44, 185-220 (2000).

Zurita-Gotor M., Blawzdziewicz J., and Wajnryb E., "Swapping trajectories: a new wall- 
induced cross-streamline particle migration mechanism in a dilute suspension of spheres," J. Fluid Mech., 592, 447-470 (2007). 


\section{List of Figures}

Figure 1 : Top : Size distribution of the particle used for the suspensions. a) Dynoseeds TS-40 $\left(d_{m}=37 \mu \mathrm{m}\right)$ and b) Dynoseeds TS-140 $\left(d_{m}=130 \mu \mathrm{m}\right)$. Bottom : Ellipticity of the particles. Smaller axis $a$ as a function of the larger axis $b$ normalized with the average particle diameter. c) Dynoseeds TS-40 $\left(d_{m}=37 \mu \mathrm{m}\right)$ and d) Dynoseeds TS-140 $\left(d_{m}=130 \mu \mathrm{m}\right)$. The average aspects ratios $a / b$ are equal to 0.924 for the TS-40 and to 0.927 for the TS-140.

Figure 2 : Rheometry: in a $50 \mathrm{~mm}$ parallel plate geometry. (Left) Viscosity of the pure fluid as a function of the shear rate $|\dot{\gamma}|$. Measurements have been performed at $T=20^{\circ} \mathrm{C}$. (Right) Viscosity of the suspension as a function of the particle volume fraction, for $|\dot{\gamma}|=20 \mathrm{~s}^{-1}$ and $T=20^{\circ} \mathrm{C}$. The continuous line corresponds to a Krieger-Dougherty law $($ Krieger $(1972)): \eta_{s}=\eta_{f}\left(1-\phi / \phi_{\max }\right)^{-2}$, with $\phi_{\max }=0.625$.

Figure 3 : Sketch of the Taylor-Couette cell.

Figure 4 : Lubrication pressure, calculated in the pure liquid of viscosity $\eta_{f}=3 \mathrm{~Pa} \cdot \mathrm{s}$, for an axis misalignment $a=100 \mu \mathrm{m}$ and for $(--) \dot{\gamma}=-100 \mathrm{~s}^{-1}\left(\Omega=-14.7 \mathrm{rad} \cdot \mathrm{s}^{-1}\right)$, $(\cdots) \dot{\gamma}=100 \mathrm{~s}^{-1},(-\cdot) P^{f}(\theta, \Omega)-P^{f}(\theta,-\Omega)$ and $(-) \lambda \cos \theta$. Note the cancellation of the lubrication pressure at fixed $\theta$ when averaged over the two directions of rotation.

Figure 5 : (- -) Ramp of shear rate $\dot{\gamma}\left(\mathrm{s}^{-1}\right)$ (right hand axis) against time $(s)$ and $(-)$ pressure transducer signal (left hand axis) measured a) behind one grid and b) (-) behind one impermeable membrane, for a suspension of particles of diameter $40 \mu \mathrm{m}$ at volume fraction $\phi \approx 0.5$.

Figure 6 : a) Image of the experimental cell with a suspension of TS-140 of volume fraction $\phi=0.45$ for $|\dot{\gamma}|=45 s^{-1}$ taken 10 seconds after the beginning of the shear. b) Spatiotemporal diagram built by plotting the light intensity of a vertical line along time (horizontal direction). The white strips on the top of the suspension correspond to a " cream" of particles that have been ejected from the suspension due to shear. 
Figure 7 : Evolution of the grid (a) and membrane (b) pressure as a function of the shear rate for a suspension with volume fraction $\phi=0.45$. ( ०) Dynoseeds TS-40, ( $\square$ ) Dynoseeds TS-140. The two dashed lines correspond to the asymptotic values of the pressure (i.e. 540 Pa for TS-140 and 1050 Pa for TS-40).

Figure 8: Evolution of the dimensionless grid (a) and membrane (b) pressure as a function of the particle volume fraction. Pressures are normalised by $\eta_{f}|\dot{\gamma}|$. (०) Dynoseeds TS-40 and ( $\square$ ) Dynoseeds TS-140. The insets display in logarithmic scale the pressures normalized with the shear stress of the suspension $\left(\eta_{s}|\dot{\gamma}|\right)$

Figure 9 : Variation of the normalized (relative to the shear stress of the pure liquid) second normal suspension stress difference $N_{2}$, with the volume fraction $\phi$. (०) Dynoseeds

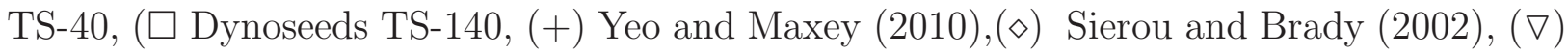

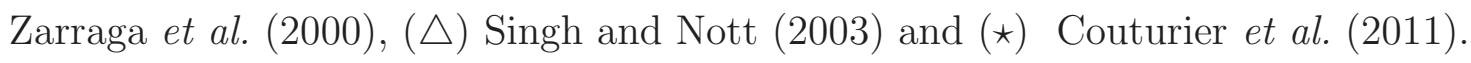

Figure $10: \Delta \Sigma_{r r}^{p}$, normalized with the suspension shear stress, as a function of $\phi(\mathrm{a})$ and of $\phi^{2}$ (b). Our data, (o) Dynoseeds TS-40 and ( $\square$ ) Dynoseeds TS-140, are compared to the data $(\diamond)$ of Sierou and Brady (2002) and the fit (solid line) of Zarraga et al. (2000) in (a), and to $y=-2 \phi^{2}$ (solid straight line) in (b).

Figure 11 : Comparison of $-\Sigma_{r r}^{p}$, normalized with the fluid shear stress, $\eta_{f}|\dot{\gamma}|$, with the theoretical prediction for the particle pressure, $\Pi / \eta_{f}|\dot{\gamma}|$, proposed by Mills (2011). 